\title{
Impact of viruses on airway diseases
}

\author{
S.L. Johnston
}

ABSTRACT: There is strong epidemiological evidence that respiratory viral infections are associated with $\mathbf{8 0 - 8 5 \%}$ of asthma exacerbations in children. There is less evidence in adults, but the available data suggest viruses are associated with around two-thirds to three-quarters of exacerbations in adults. These associations include severe exacerbations requiring hospitalisation. The most common viruses detected in these studies were rhinoviruses, accounting for two-thirds of viruses detected.

Asthmatics have increased susceptibility to respiratory virus infection and have recently been shown to have profoundly defective interferon- $\beta$ responses to virus infection, resulting in increased virus replication.

Atypical bacterial infections are also associated with chronic asthma and asthma exacerbations and a recent study indicates antibiotic therapy active against atypical bacteria is effective in treatment of exacerbations. Recent data also indicates asthmatics are at increased risk of invasive pneumococcal disease, suggesting they may also have impaired antibacterial immunity.

Research is urgently required to determine whether augmenting anti-infective immunity is beneficial in the treatment/prevention of asthma exacerbations. More recent data also implicates viruses in the majority of exacerbations of chronic obstructive pulmonary disease. Studies are also required investigating anti-infective host defence in chronic obstructive pulmonary disease.

KEYWORDS: Asthma, chronic obstructive pulmonary disease, host defence, respiratory virus infection

$\mathbf{R}$ espiratory tract infections are the most common infections to afflict mankind and are responsible for an enormous burden of disease, ranging from trivial mild common colds, to severe fatal pneumonias. All known respiratory viruses are able to produce the illness complex recognised as the common cold. It has also long been recognised that respiratory viruses associated with upper respiratory infections, such as influenza, parainfluenza, respiratory syncytial virus (RSV) and adenoviruses are also frequently associated with lower respiratory tract infections. Although there is a good deal of overlap in the disease spectra associated with these viruses, adenoviruses are classically associated with severe destructive pneumonias, influenza with acute bronchitis and pneumonia, parainfluenza viruses with laryngotracheobronchitis and RSV with acute bronchiolitis.

Rhinoviruses are the commonest pathogens of the respiratory tract. Their identification was achieved only 4 decades ago; however, they are the cause of the majority of common colds, a disease affecting all human populations and causing considerable morbidity and an enormous economic burden worldwide [1, 2]. Nevertheless, probably due to the benign nature of most rhinovirus colds, little attention has been paid to these viruses until recent years, when they have been implicated in acute exacerbations of asthma and chronic obstructive pulmonary disease (COPD). Several early studies sought to objectively establish a link between virus infections and asthma exacerbations [3-5]; however, it was only after the advent of PCR-based detection methodologies that it was demonstrated that the vast majority of asthma exacerbations in children [6] and between half to three-quarters in adults $[7,8]$ are associated with respiratory viral infections. In these studies, rhinoviruses were the most frequently identified pathogen.

These important epidemiological observations have raised considerable interest in the underlying mechanisms of virus-induced asthma exacerbations, the understanding of which could lead to new strategies for treatment. Recent studies have also suggested virus infections are a major cause of acute exacerbations of COPD [9, 10]. This article will briefly review the clinical and epidemiological evidence linking virus infections with acute exacerbations of lower respiratory disease.

The epidemiological data linking viruses with exacerbations of airway disease has fuelled
CORRESPONDENCE

S.L. Johnston

Dept of Respiratory Medicine

National Heart and Lung Institute Imperial College London

Norfolk Place

London W2 1 PG

UK

Fax: 442072628913

E-mail: s.johnston@imperial.ac.uk 
interest in the mechanisms of host defence against virus infection. A recent study has demonstrated deficient innate antiviral immunity in asthmatics [11]; this review will conclude with discussion of these exciting findings and the implications they have for the treatment and prevention of virus-induced exacerbations of airway disease.

\section{CLINICAL AND EPIDEMIOLOGICAL EVIDENCE Viruses and asthma exacerbations}

A large number of epidemiological studies on the role of viruses in asthma exacerbations were performed during the 1970s and 1980s [12]. Incidental studies attempted to isolate viruses in acute episodes of asthma, while prospective studies followed up patients with asthma and virus isolation was undertaken either during respiratory infections or on a regular basis. Several important conclusions were drawn from these early reports. First, while rhinoviruses were among the most prevalent microorganisms in the majority of cases, variability in their identification rates was observed between the studies and the overall identification rates correlated with the success or lack of success of rhinovirus detection. This strongly suggested deficiencies in rhinovirus detection methodology. Secondly, it was shown that early sampling is critical in the successful isolation of viruses [4], and that identification rates tend to be higher in outpatients or subjects from general practice than hospitalised patients and in prospective rather than incidental studies. Finally, only a few studies focused on adults, showing considerably lower overall and rhinovirus-specific identification rates than those obtained in children.

The advent of PCR-based methods for virus identification proved to be a turning point in the understanding of asthma exacerbation pathogenesis. This was particularly true for rhinoviruses and coronaviruses, for which classical methods are technically demanding and of low sensitivity [13, 14]. Recent studies using PCR for detection of rhinoviruses, and more recently other viruses as well, demonstrate that virus involvement in asthma exacerbations in children is much more frequent than previously believed, with virus detection rates of between $80-86 \%[6,15-18]$. In all these studies rhinoviruses were the predominant pathogens, being detected in $\sim 50 \%$ of exacerbations.

A study in adults was performed by NicHOLSON et al. [7], in which 138 asthmatic adults, aged 19-46 yrs, were followed up during a 2-yr period. Virus identification rates were lower, associated with $44 \%$ of asthma exacerbations. However, when subjective criteria were used, $80 \%$ of asthma exacerbations occurred with symptomatic colds, while $90 \%$ of colds were associated with symptoms of asthma. It is possible that the discrepancy between subjective symptomatology and objective virus detection may be attributed to a decreased virus shedding in adults [7], or perhaps to less intensive monitoring and later sampling than was the case in the studies in children. This interpretation is supported by a recent study in which viruses were detected in sputum samples from adults attending the emergency department with acute asthma exacerbations. In this study viruses were detected in $76 \%$ of samples, and virus-induced lower airway cytotoxicity was the major predictor of severity of exacerbation [8]. A more recent study using similar methodology had a virus detection rate in sputum of $70 \%$ in adults attending the emergency room with an asthma exacerbation [18].

Studies of severe asthma requiring hospitalisation also suggest virus infections are the major determinant of risk and identify striking seasonal patterns of admissions entirely consistent with a viral aetiology. These seasonal patterns also identify epidemics of admissions that would be suitable for intervention with preventive therapies. Virus detection data obtained from a Southampton paediatric cohort where compared with hospitalisations for asthma in the same area, during the same time period. Strong correlations were found between the seasonal patterns of upper respiratory infections and hospital admissions for asthma [19]. These included both paediatric, adult and combined admissions, though they were stronger for the paediatric population. Interestingly, there was a close relationship between the peaks of both respiratory infections and hospital admissions and the start of a new school-term or half-term, establishing school attendance as the major factor determining admissions for asthma. These finding have recently been replicated in Canada using a similar approach [20].

These results confirm that the great majority of asthma exacerbations are associated with viral respiratory tract infections in both adults and children and that the great majority of viruses associated are rhinoviruses. These results are summarised in table 1.

\section{Role of respiratory viruses in COPD exacerbations}

COPD is a common, chronic disease affecting $4 \%$ of males and $2 \%$ of females aged $>45$ yrs. Its prevalence is increasing and it is a significant cause of mortality and morbidity and an enormous consumer of healthcare costs. Around 70\% of these costs are due to hospitalisation during acute exacerbations of COPD, which cause considerable morbidity and have a mortality of $\sim 15 \%$. There is increasing evidence that viruses can cause exacerbations of COPD. Earlier studies detected viruses in $15-28 \%$ of COPD exacerbations [21, 22], but used limited methodology for virus detection in that they did not include PCR.

A more recent study has examined the importance of respiratory viral infections in COPD exacerbations using PCR [9]. A total of 83 COPD patients recorded daily, any increases in respiratory symptoms and changes in peak expiratory flow (PEF). Patients were seen at baseline and at acute exacerbation. Nasopharyngeal samples were taken for virus culture and PCR and blood samples for serology. Altogether, 77 viruses were detected in $66(39.2 \%)$ of 168 COPD exacerbations and of these, $39(58.2 \%)$ were rhinoviruses.

Viral exacerbations were associated with increased dyspnoea, a higher total symptom count at presentation, longer median symptom recovery period of 13 days and a slower rate of symptom return to baseline [9]. During viral exacerbations there was a tendency toward greater falls in PEF, higher plasma fibrinogen and greater serum interleukin-6 levels. Therefore, virus-associated exacerbations showed greater symptomatic changes with increased systemic inflammatory markers. Thus, prevention of viral infections may lead to a decrease in both exacerbation frequency and morbidity associated with COPD. 
TABLE 1 Summary of studies investigating virus infections in exacerbations of asthma using modern molecular detection methods

\begin{tabular}{|c|c|c|c|c|}
\hline Overall detection rate \% & $\begin{array}{l}\text { Proportion of exacerbations } \\
\text { due to rhinoviruses \% }\end{array}$ & Age of subjects & Setting & Reference \\
\hline 83 & 71 & $2-16$ yrs & Emergency room & RAKES [15] \\
\hline 80 & 47 & $2-14$ yrs & Hospitalised & FREYMUTH [16] \\
\hline 78 & 46 & $7-11$ yrs & Community & CHAUHAN [17] \\
\hline 76 & 11 & Adults & Emergency room & WARK [8] \\
\hline 44 & 26 & Adults & Community & NiCHOLSON [7] \\
\hline
\end{tabular}

\#: this figure is for adults and children combined; data not given separately.

This study directly implicates virus infections in $\sim 40 \%$ of COPD exacerbations; however, it is likely that this figure is an underestimate of the true association. The reason for this is that the subjects in the above study provided upper respiratory samples only, and these were provided at the time the exacerbation of their lower respiratory disease occurred. It is well recognised that in COPD, upper respiratory symptoms may precede the onset of the exacerbation by several days.

As a result of this, virus yields in upper respiratory samples taken at the time of exacerbation of lower respiratory tract disease are likely to have been low, as the sample may have been taken at a time when the upper respiratory infection was over in a substantial number of cases. This interpretation is supported by prospective diary card data collected in the above study, which demonstrated that $65 \%$ of patient had common cold symptoms prior to their exacerbation.

A more recent study has reported the finding of viruses in $56 \%$ of nasal and/or sputum samples in a study of patients admitted to hospital with COPD exacerbations in Germany [10].

Further studies on the role of virus infections in COPD exacerbations are clearly needed, but the above data (table 1) strongly suggests that the contribution of viruses and of rhinoviruses in particular is not likely to be less important than the established role of these viruses in asthma exacerbations.

\section{MECHANISMS}

\section{Host defence against virus infection in the lower airways}

Recent work indicates that asthmatic individuals are more susceptible to naturally occurring rhinovirus infection than normal individuals in that when infected, lower respiratory tract symptoms and changes in PEF were both more severe and of longer duration in the asthmatic than in the normal subjects [23]. The reasons for this increased susceptibility in asthmatics are largely unknown.

The airway epithelial cell is known to contribute to antiviral and inflammatory immune responses through the production of a wide range of cytokines, chemokines and other mediators. The type 1 interferons (IFNs), (the IFN- $\alpha$ s (of which 13 are recognised) and IFN- $\beta$ ) are induced by viral infection of the host cell. IFN- $\beta$ is induced first, and it then induces more of itself and the IFN- $\alpha$ s in both autocrine and paracrine manners, thereby establishing an antiviral state, characterised by the expression and antiviral activity of IFN-stimulated genes.

In addition to the antiviral activity of type 1 IFNs, rapid induction of apoptosis following virus infection of a cell is also a critical mechanism for the limitation of viral replication. Recent work has demonstrated a link between IFN- $\alpha / \beta$ signalling, the transcriptional activation of p53 and the induction of its proapoptotic target genes in virally infected cells [24]. This induction subsequently results in the activation of apoptosis leading to programmed cell death. If programmed cell death occurs within a few hours of virus infection of a cell, that cell is not available for successful virus replication, which therefore aborts. In addition, the infected cell is phagocytosed as a result of undergoing apoptosis, rather than dying by cell necrosis as a result of virus replication. Phagocytosis will therefore remove infected cells without stimulating inflammatory pathways, while death by necrosis, in addition to releasing new viruses to infect other cells, will also result in release of many mediators of inflammation, leading to a robust inflammatory response to infection.

WARK et al. [11] recently investigated IFN- $\beta$ induction by rhinovirus in primary bronchial epithelial cells from atopic asthmatic and nonatopic normal individuals in vitro. A profound deficiency in IFN- $\beta$ induction in the asthmatic cells was observed, accompanied by impaired apoptosis and increased viral replication (fig. 1). These studies identify a novel mechanism for increased susceptibility to infection in asthmatics and suggest that efforts to augment innate immune responses could provide protection against virus-induced asthma exacerbations in asthma.

Asthma is associated with T-helper cell (Th)2-biased acquired immune responses, and PAPADOPOULOS et al. [25] have reported data indicating that asthmatics have impaired peripheral blood Th1-acquired immune responses to respiratory virus infection. Further studies are needed to determine whether asthmatics have impaired Th1-acquired immune responses in the respiratory tract. 
a)

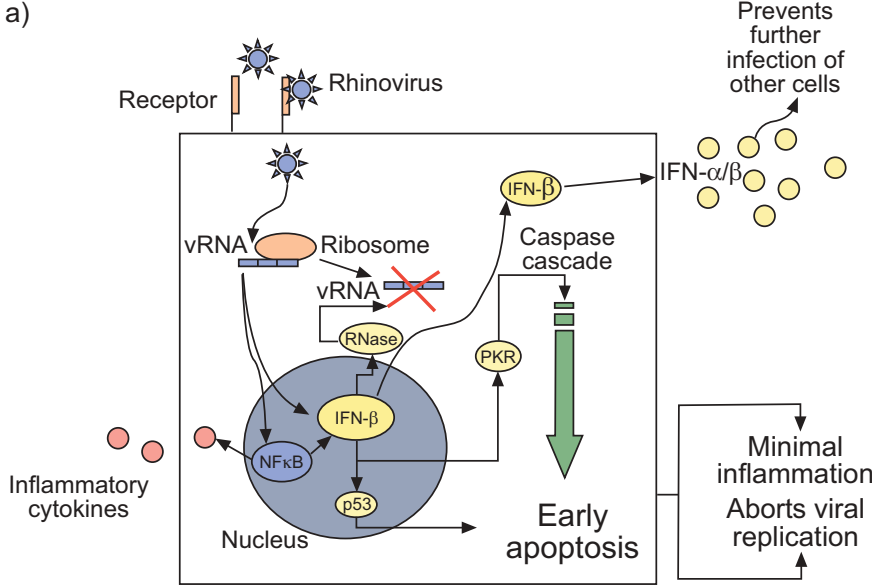

b)

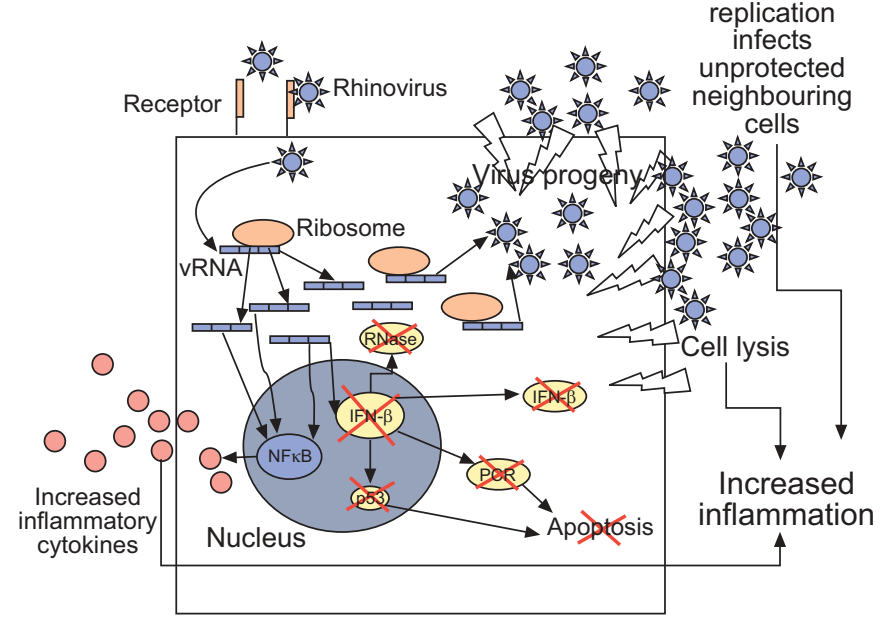

FIGURE 1. Virus infection in a) normal and b) asthmatic bronchial epithelial cells. In normal bronchial epithelial cells, virus infection leads to rapid induction of IFN- $\beta$. This induces more of itself, as well as the $\alpha$-IFNs, to induce an antiviral state in the infected cell and in neighbouring cells. This antiviral state includes production of RNases to digest viral RNA and inhibit viral replication, and p53 and protein kinase $\mathrm{R}$ to induce rapid induction of apoptosis. Apoptosis denies the virus access to cellular biochemical pathways required for replication and effectively aborts virus replication, nuclear factor (NF) $\mathrm{kB}$ induced inflammatory cytokine production is minimised and the apoptotic cell is phagocytosed. These combined activities virtually prevent virus replication and effectively limit virus induced inflammation. In contrast, when an asthmatic cell is infected, IFN- $\beta$ production is profoundly impaired and antiviral pathways are either not induced or only weakly so. Virus replication therefore proceeds unchecked by RNases, or by apoptosis. Effective replication ensues, strongly inducing NFKB and inflammatory cytokine production. Eventually, the heavily infected cell necroses and lyses, releasing further viruses to infect neighbouring cells, which are not protected by IFNs- $\alpha / \beta$. The outcome is a high virus load unchecked by antiviral responses and therefore severe inflammation. IFN: interferon; PKR: protein kinase R; NFкB: nuclear factor-кB.

Although the remit of this article is to discuss viral infections, it is pertinent also to mention two very interesting recent studies relating to bacterial infections. A number of studies (reviewed in [26]) suggest a role for atypical bacterial (Chlamydia pneumoniae and Mycoplasma pneumoniae) infection as a co-factor in both chronic-stable asthma and acute exacerbations of asthma. A recent study has reported that antibacterial therapy active against atypical bacteria is of therapeutic benefit in the treatment of acute exacerbations of asthma [27]. This study was unable to determine whether efficacy was related to activity against atypical bacterial infection, or to anti-inflammatory or possibly even antiviral activity. Further studies are clearly required to confirm these interesting results and to investigate the mechanism(s) of therapeutic benefit. Asthmatics have also very recently been reported to have an increased risk of invasive pneumococcal infections [28]. This data has important implications for public health policy relating to pneumococcal vaccination, but it also, along with the data on atypical bacterial infection, has very interesting implications regarding host immunity in asthmatics, suggesting that they may have impaired immunity to both viral and bacterial infection.

\section{CONCLUSIONS}

The association of respiratory virus infections with asthma exacerbations has been clearly demonstrated in a number of studies, in both adults and children. Asthmatics have increased susceptibility to virus infections, and have also been reported to have increased risk of severe bacterial infections. Asthmatics have impaired innate immune responses to virus infections and may also have impaired Th1-acquired immunity. Given their increased risk of severe bacterial infections and the recent evidence that antibacterial therapy is of therapeutic benefit in acute exacerbations of asthma, further studies examining the role of both viral and bacterial infections, and of host defence in exacerbations of asthma, are now required. The available evidence suggests that research into ways of augmenting antiinfective immunity in asthma might be rewarding.

Further studies will be necessary to evaluate the role of viruses in exacerbations of chronic obstructive pulmonary disease; however, the evidence available to date suggests that viruses are associated with around two-thirds to three-quarters of exacerbations, perhaps sometimes in concert with bacterial infections. There is no information available regarding whether chronic obstructive pulmonary disease patients have impaired antiviral or antibacterial immunity; such studies are urgently required.

\section{REFERENCES}

1 Papadopoulos NG, Johnston SL. Rhinoviruses. In: Zuckerman A, Banatvala J, Pattison J, eds. Principles and Practice of Clinical Virology, 4th ed. Chichester, John Wiley \& Sons, 2000: 329-343.

2 Tyrrell DAJ. A collaborative study of the aetiology of acute respiratory infections in Britain 1961-4. A report of the Medical Research Council Working Party on acute respiratory virus infections. BMJ 1965; 2: 319-326.

3 Mertsola J, Ziegler T, Ruuskanen O, Vanto T, Koivikko A, Halonen P. Recurrent wheezy bronchitis and viral respiratory infections. Arch Dis Child 1991; 66: 124-129.

4 Horn M, Brain E, Gregg I, Yealland S, Inglis J. Respiratory viral infection in childhood. A survey in general practice, Roehampton 1967-1972. J Hyg (Lond) 1975; 74: 157-168.

5 Kellner G, Popow-Kraupp T, Kundi M, Binder C, Kunz C. Clinical manifestations of respiratory tract infections due to respiratory syncytial virus and rhinoviruses in hospitalized children. Acta Paediatr Scand 1989; 78: 390-394. 
6 Johnston SL, Pattemore PK, Sanderson G, et al. Community study of role of viral infections in exacerbations of asthma in 9-11 year old children. BMJ 1995; 310: 1225-1228.

7 Nicholson KG, Kent J, Ireland DC. Respiratory viruses and exacerbations of asthma in adults. BMJ 1993; 307: 982-986.

8 Wark PA, Johnston SL, Moric I, Simpson JL, Hensley MJ, Gibson PG. Neutrophil degranulation and cell lysis is associated with clinical severity in virus-induced asthma. Eur Respir J 2002; 19: 68-75.

9 Seemungal T, Harper-Owen R, Bhowmik A, et al. Respiratory viruses, symptoms, and inflammatory markers in acute exacerbations and stable chronic obstructive pulmonary disease. Am J Respir Crit Care Med 2001; 164: 1618-1623.

10 Rohde G, Wiethege A, Borg I, et al. Respiratory viruses in exacerbations of chronic obstructive pulmonary disease requiring hospitalisation: a case-control study. Thorax 2003; 58: $37-42$.

11 Wark PA, Johnston SL, Bucchieri F, et al. Asthmatic bronchial epithelial cells have a deficient innate immune response to infection with rhinovirus. J Exp Med 2005; 201: 937-947.

12 Pattemore PK, Johnston SL, Bardin PG. Viruses as precipitants of asthma symptoms. I. Epidemiology. Clin Exp Allergy 1992; 22: 325-336.

13 Johnston SL, Sanderson G, Pattemore PK, et al. Use of polymerase chain reaction for diagnosis of picornavirus infection in subjects with and without respiratory symptoms. J Clin Microbiol 1993; 31: 111-117.

14 Myint S, Johnston S, Sanderson G, Simpson H. Evaluation of nested polymerase chain methods for the detection of human coronaviruses 229E and OC43. Mol Cell Probes 1994; 8: 357-364.

15 Rakes GP, Arruda E, Ingram JM, et al. Rhinovirus and respiratory syncytial virus in wheezing children requiring emergency care. IgE and eosinophil analyses. Am J Respir Crit Care Med 1999; 159: 785-790.

16 Freymuth F, Vabret A, Brouard J, et al. Detection of viral, Chlamydia pneumoniae and Mycoplasma pneumoniae infections in exacerbations of asthma in children. J Clin Virol 1999; 13: 131-139.

17 Chauhan AJ, Inskip $\mathrm{HM}$, Linaker $\mathrm{CH}$, et al. Personal exposure to nitrogen dioxide (NO2) and the severity of virus-induced asthma in children. Lancet 2003; 361 1939-1944.

18 Grissell TV, Powell H, Shafren DR, et al. IL-10 Gene expression in acute virus-induced asthma. Am J Respir Crit Care Med 2005; 172: 433-439.

19 Johnston SL, Pattemore PK, Sanderson G, et al. The relationship between upper respiratory infections and hospital admissions for asthma: a time-trend analysis. Am J Respir Crit Care Med 1996; 154: 654-660.

20 Johnston NW, Johnston SL, Duncan JM, et al. The September epidemic of asthma exacerbations in children: a search for etiology. J Allergy Clin Immunol 2005; 115: 132-138.

21 Smith CB, Golden CA, Kanner RE, Renzetti AD. Association of viral and Mycoplasma pneumoniae infections with acute respiratory illness in patients with chronic obstructive pulmonary diseases. Am Rev Respir Dis 1980; 121: 225-232.

22 Greenberg SB, Allen M, Wilson J, Atmar RL. Respiratory viral infections in adults with and without chronic obstructive pulmonary disease. Am J Respir Crit Care Med 2000; 162: 167-173.

23 Corne JM, Marshall C, Smith S, et al. Frequency, severity, and duration of rhinovirus infections in asthmatic and non-asthmatic individuals: a longitudinal cohort study. Lancet 2002; 359: 831-834.

24 Takaoka A, Hayakawa S, Yanai $\mathrm{H}$, et al. Integration of interferon-alpha/beta signalling to p53 responses in tumour suppression and antiviral defence. Nature 2003; 424: 516-523.

25 Papadopoulos NG, Stanciu LA, Papi A, Holgate ST, Johnston SL. A defective type 1 response to rhinovirus in atopic asthma. Thorax 2002; 57: 328-332.

26 Johnston SL, Martin RJ. Chlamydophila pneumoniae and Mycoplasma pneumoniae: a role in asthma pathogenesis? Am J Respir Crit Care Med 2005 (Epub ahead of print).

27 Johnston SL, Blasi F, Black PN, Martin RJ, Farrell DJ, Nieman RB. The effect of Telithromycin in acute exacerbations of asthma: the TELICAST study (Abstract). Am J Respir Crit Care Med 2005; 2: A569.

28 Talbot TR, Hartert TV, Mitchel E, et al. Asthma as a risk factor for invasive pneumococcal disease. $N$ Engl J Med 2005; 352: 2082-2090. 